\title{
A systematic review of the factors predicting the interest in cosmetic plastic surgery
}

\author{
Panagiotis Milothridis ${ }^{1,2}$, Leonidas Pavlidis², Anna-Bettina Haidich ${ }^{1}$, Efharis Panagopoulou ${ }^{1}$ \\ ${ }^{1}$ Laboratory of Hygiene, ${ }^{2}$ Department of Plastic Surgery, Medical School, Aristotle University of Thessaloniki, \\ Thessaloniki, Greece
}

Address for correspondence: Dr. Panagiotis Milothridis, Anthokipwn 64, Euosmos, Thessaloniki, Greece. E-mail: milothridis@gmail.com

\section{ABSTRACT}

Background: A systematic review of the literature was performed to clarify the psychosocial characteristics of patients who have an interest in cosmetic plastic surgery. Methods: Medical literature was reviewed by two independent researchers, and a third reviewer evaluated their results. Results: Twelve studies addressing the predictors of interest in cosmetic surgery were finally identified and analysed. Interest in cosmetic surgery was associated with epidemiological factors, their social networks, their psychological characteristics, such as body image, self-esteem and other personality traits and for specific psychopathology and found that these may either positively or negatively predict their motivation to seek and undergo a cosmetic procedure. Conclusions: The review examined the psychosocial characteristics associated with an interest in cosmetic surgery. Understanding cosmetic patients' characteristics, motivation and expectation for surgery is an important aspect of their clinical care to identify those patients more likely to benefit most from the procedure.

\section{KEY WORDS}

Cosmetic surgery; predictors of interest; psychosocial factors

\section{INTRODUCTION}

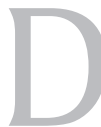

espite the financial recession of the last decade, the number of people seeking a cosmetic plastic surgery procedure has increased. This trend can also be attributed to the outbreak of minimally invasive procedures (e.g., Botox and fillers injections) which has become very popular among cosmetic patients. ${ }^{[1]}$ Specifically, 15.6 million cosmetic procedures, including both minimally invasive and surgical, were performed

\begin{tabular}{|l|l|}
\hline \multicolumn{2}{|c|}{ Access this article online } \\
\hline Quick Response Code: & Website: \\
\hline & www.ijps.org \\
\cline { 2 - 2 } & Dol: \\
\hline
\end{tabular}

in the United States in 2014, an increase of 3\% since 2013, which reflects a total cost of $\$ 12.9$ billion. ${ }^{[2]}$ The above figures highlight the fact that cosmetic surgery is a recession-proof industry.

However, this increase in cosmetic surgery also highlights the need to investigate the motives of cosmetic patients to choose the patients who will benefit from the aesthetic procedures. Because of the fact that cosmetic

This is an open access article distributed under the terms of the Creative Commons Attribution-NonCommercial-ShareAlike 3.0 License, which allows others to remix, tweak, and build upon the work non-commercially, as long as the author is credited and the new creations are licensed under the identical terms.

For reprints contact: reprints@medknow.com

How to cite this article: Milothridis $P$, Pavlidis $L$, Haidich $A B$, Panagopoulou E. A systematic review of the factors predicting the interest in cosmetic plastic surgery. Indian J Plast Surg 2016;49:397-402. 
plastic surgery is an elective kind of operation and the possible candidates should be carefully selected, the characteristics of people seeking a surgical improvement of their appearance have to be thoroughly clarified. Studies have examined the psychosocial profile of people seeking an elective cosmetic surgery. This is clinically relevant because some special psychosocial characteristics may predispose patients for some complications that follow aesthetic surgery. For example, it has been shown that the majority of body dysmorphic patients are dissatisfied about the plastic surgery result and resentful towards their surgeons. ${ }^{[3]}$

Given the fact that there is no systematic information about the psychosocial characteristics of people seeking specific types of cosmetic surgery, we decided to conduct a systematic review of the literature to examine the psychosocial characteristics associated with different types of cosmetic surgery. This information could then be used to develop also a screening instrument for clinical use.

\section{METHODS}

A systematic review ${ }^{[4.7]}$ of the medical literature was conducted by two independent researchers. A third independent reviewer evaluated their results.

Initially, we formed a research protocol with clearly defined research questions to eliminate selection bias of the study. The protocol was based on the guidelines of PRISMA statement. ${ }^{[5]}$ Any future alteration in the original protocol would be declared by the authors along with the reason that led to its modification.

The PubMed/MEDLINE databases were searched using the terms 'psychology', 'characteristics', 'interest_in_plastic_surgery' and 'seeking_ cosmetic_surgery'. Furthermore, we collected studies from the reference lists of the selected articles. There were no publication year restrictions. The date of the last search was $20^{\text {th }}$ August, 2015. The review included case-control, case series and cross-sectional studies, which examine theoretical interest in cosmetic plastic surgery identified by questionnaires and phone interviews as research tools. We included only published papers regardless of the country of origin written in English. Exclusion criteria were reviews and expert opinions, as well as case studies with fewer than twenty participants.

\section{RESULTS}

Our literature search identified 12 studies [Table 1] addressing the predictors of interest for cosmetic surgery [Figure 1].

\section{Factors predicting the interest in seeking a cosmetic procedure Epidemiological factors}

One study examines the influence of gender on the interest in cosmetic plastic surgery. Frederick et al. ${ }^{[18]}$ analysed data from 25,714 men and 26,963 women and found that half of the women (48\%) were interested in one or more cosmetic procedures, while one fourth $(23 \%)$ of the men reported such interest. No significant differences were shown in regard to age, even though older women showed greater certainty in their decision to undergo plastic surgery. ${ }^{[18]}$ Moreover, age was used as a controlling variable in logistic regression analysis models in another study. ${ }^{[17]}$ Body mass index (BMI) was found to not correlate with the general interest in plastic surgery, yet it was an independent predictor in certain procedures, as in liposuction, regarding both men and women. ${ }^{[18]}$ Similarly, Cook et al. ${ }^{[11]}$ found that women who decided to undergo a breast augmentation mammoplasty tend to be thinner than the general population and this is consistent with the findings by Javo and Sørlie ${ }^{[19]}$ who proved that distorted eating behaviour is a significant positive predictor of interest in plastic surgery (odds ratio $[\mathrm{OR}]=4.09$; $P<0.001)$ in the univariate model. Cook et al. ${ }^{[11]}$ also examined alcohol consumption as a predicting factor for someone's decision to undergo breast augmentation and found that women in the cosmetic group were more likely to drink a greater average number of drinks per week $(\mathrm{OR}=2.9)$, while there was no correlation with smoking. Finally, education was proved to be a strong negative predictor for someone's general interest in plastic surgery $(\mathrm{OR}=0.57 ; P<0.001) .^{[19]}$

\section{Social networks}

Our review identified five studies ${ }^{[11,14,15,17,19]}$ examining the impact of social networks on interest in cosmetic plastic surgery. Specifically, high quality of relationship with parents has been found to be a negative predictor of interest in cosmetic surgery both in the univariate $(\mathrm{OR}=0.87$; 95\% confidence interval $[\mathrm{CI}] ; 0.83-0.91: P<0.001)$ and multiple logistic regression analyses $(\mathrm{OR}=0.94$; 95\% CI; 0.88-0.99: $P=0.018) .^{[19]}$ Marital status was found to be a significant covariate indicating that fewer women willing to undergo cosmetic surgery were 
Table 1: Studies included in this review

\begin{tabular}{|c|c|c|c|c|c|}
\hline $\begin{array}{l}\text { Authors } \\
\text { (references) }\end{array}$ & Year & Procedure & Study type & $\begin{array}{l}\text { Number of } \\
\text { patients }\end{array}$ & Factors predicting interest \\
\hline Wright and Wright ${ }^{[8]}$ & 1975 & Rhinoplasty & Case series & 40 & Personality characteristics, psychopathology \\
\hline Napoleon ${ }^{[9]}$ & 1993 & Various & Prospective & 133 & Personality disorders \\
\hline Dunofsky ${ }^{[10]}$ & 1997 & Face surgery & Case-control & 36 & Personality disorders \\
\hline Cook et al. ${ }^{[11]}$ & 1997 & $\begin{array}{l}\text { Breast } \\
\text { augmentation }\end{array}$ & Case-control & 80 & $\begin{array}{l}\text { Number of lifetime sexual partners, age at first pregnancy, } \\
\text { terminated pregnancies, use of oral contraceptives, } \\
\text { alcohol consumption, smoking, body mass index }\end{array}$ \\
\hline Pertschuk et al. ${ }^{[12]}$ & 1998 & Various & Case-control & 30 & Body image, gender \\
\hline Ishigooka et al. ${ }^{[13]}$ & 1998 & Various & Retrospective & 415 & Personality disorders \\
\hline Didie and Sarwer ${ }^{[14]}$ & 2003 & $\begin{array}{l}\text { Breast } \\
\text { augmentation }\end{array}$ & Case-control & 30 & $\begin{array}{l}\text { Body image } \\
\text { Sociocultural influence, marital/sexual satisfaction }\end{array}$ \\
\hline Sarwer et al..$^{[15]}$ & 2003 & $\begin{array}{l}\text { Breast } \\
\text { augmentation }\end{array}$ & Case-control & 30 & Body image, teasing history \\
\hline Ferraro et al..$^{[16]}$ & 2005 & Various & Case-control & 70 & Self-esteem \\
\hline Von Soest et al. ${ }^{[17]}$ & 2006 & Various & Case-control & 1102 & $\begin{array}{l}\text { Being recommended cosmetic surgery, knowing someone } \\
\text { who had cosmetic surgery, teasing history, body image, } \\
\text { self-esteem, self-monitoring, marital status }\end{array}$ \\
\hline Frederick et al..$^{[18]}$ & 2007 & Various & Cross-sectional & 52677 & Body image, age, gender \\
\hline Javo and Sørlie ${ }^{[19]}$ & 2009 & Various & Cross-sectional & 1880 & $\begin{array}{l}\text { Personality characteristics, body dimorphic disorder-like } \\
\text { symptoms, self-esteem, body image, satisfaction with } \\
\text { sexual life, distorted eating behaviour, education, teasing } \\
\text { history, knowing someone who had cosmetic surgery, being } \\
\text { recommended one, having children, emotional distress }\end{array}$ \\
\hline
\end{tabular}

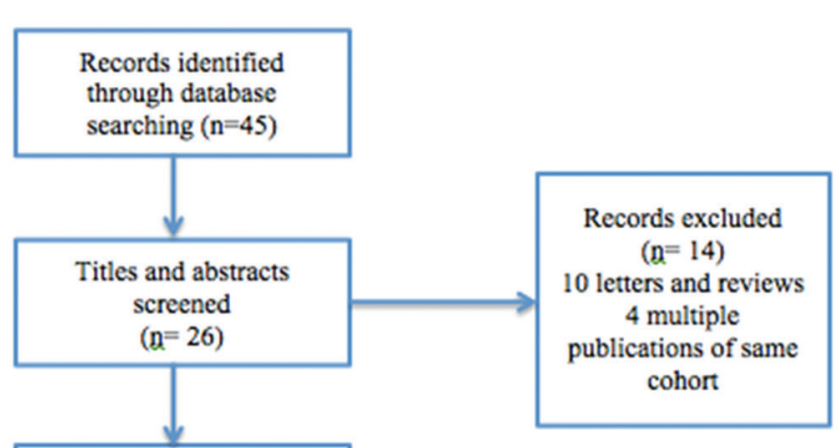

Studies included for data extraction $(n=12)$

Figure 1: Flow diagram

married compared with those not wishing to undergo surgery $(\mathrm{OR}=0.47 ; P<0.01) .{ }^{[17]}$ On the other hand, having children has been found to be a predictor of an interest in cosmetic surgery $(\mathrm{OR}=1.70$ in multivariate regression analysis; $P<0.001) .^{[19]}$ Finally, Cook et al. ${ }^{[11]}$ found that women who have undergone breast augmentation mammoplasty have had a greater lifetime number of sexual partners (prevalence $\mathrm{OR}[\mathrm{POR}]=8.9$ ), were younger at first pregnancy ( $\mathrm{pOR}=1.6$ ), are more likely to have a history of terminated pregnancies $(\mathrm{pOR}=2.0)$ and to the use of oral contraceptives ( $\mathrm{POR}=2.2$ ).

Social norms are also associated with cosmetic surgery. ${ }^{[14]}$ Von Soest et al. ${ }^{[17]}$ have shown that an interest in cosmetic surgery is strongly predicted by knowing someone who have undergone cosmetic surgery $(\mathrm{OR}=2.89 ; P<0.01)$. Moreover, people who have been teased about their physical appearance in childhood or adolescence have a tendency in seeking cosmetic surgery as proposed by Sarwer et al. ${ }^{[15]}$ and this has been further hypothesised and proven by Von Soest et al. ${ }^{[17]}$

\section{Body image, self-esteem and other personality traits}

Sarwer et al. ${ }^{[15]}$ assumed that someone with high body image orientation and low body image evaluation is more likely to choose cosmetic plastic surgery. This hypothesis has not been confirmed by other studies finding no difference in global body image between the cosmetic and the control groups. ${ }^{[12]}$ In two studies, ${ }^{[17,18]}$ body image evaluation rated higher among women who were not motivated to undergo cosmetic surgery while women who had an interest in such procedures showed statistically significant higher body image orientation. Even though results about the correlation between global body image and general motivation in cosmetic surgery are ambiguous, it is quite clear that someone's interest in a cosmetic procedure is related with dissatisfaction about the specific body element he/she wishes to alter.

Despite the fact that someone would expect that low self-esteem could be a motive to undergo plastic surgery, 
our systematic review could not identify any study which proves such correlation. ${ }^{[10,16,17,19]}$

Javo and Sørlie found that personality dispositions appear to be independent and stable predictors of health-related habits and attitudes in a variety of contexts. In particular, except for extraversion, all the big five personality traits, i.e. agreeability, conscientiousness, emotional stability and openness were significant negative predictors of an interest in cosmetic surgery in the univariate analyses $(P<0.001) .^{[19]}$ Moreover, Von Soest et al. concluded that self-monitoring, another personality trait which indicates the ability someone could regulate their behaviour to their social environment, is related to higher probability of deciding to undergo cosmetic surgery. ${ }^{[17]}$ The so-called high self-monitorers are persons who regulate their behaviour in accordance with situational cues, whereas low self-monitorers' behaviour depends more on their inner attitudes, emotions and dispositions. ${ }^{[20,21]}$

\section{Psychopathology}

It is undisputable that body dysmorphic disorder is common among people who have an interest in plastic surgery, accounting to a prevalence of $15 \%$, while it does not exceed $1 \%-2 \%$ in the general population. ${ }^{[3]}$ Indeed, Javo and Sørlie found that body dysmorphic disorder-like symptoms were a strong predictor $(\mathrm{OR}=2.31)$ for an interest in cosmetic surgery. ${ }^{[19]}$ Narcissistic personality disorder is defined by someone's grandiosity, need for admiration and lack of empathy. It can be found in $25 \%$ of people seeking cosmetic surgery, ${ }^{[10,13]}$ especially rejuvenation procedures. Similarly, $3.4 \%$ of people who are motivated to undergo a cosmetic surgery are characterised by the histrionic personality disorder with emotional excess and the need to gain the attention of others, while other authors raise the percentage of histrionic personality prevalence among cosmetic patients up to $9.7 \% .{ }^{[9,13]}$

\section{DISCUSSION}

Our systematic literature review identified 12 studies concerning the psychosocial characteristics of people who have an interest in plastic surgery. We focused on the theoretical interest and not the ultimate decision to undergo a cosmetic procedure since it is deprived of factors, such as fear of complications or the ability to afford the procedure. However, we believe that future studies should be conducted to clarify the factors that influence the gap between plain interest in cosmetic surgery and the decision to undergo one.

Our review showed no relationship between age and choice of cosmetic surgery. Although biological ageing is an inevitable process, there have been medical advances in cosmetic plastic surgery, producing numerous options to slow and to reverse the ageing process. Indeed, it has been shown that people undergoing facelift procedures seem to be $5.7-8.3$ years younger post-surgery. ${ }^{[22]}$ That is the reason why we expect older people to be more interested in seeking plastic surgery procedures in general. Nevertheless young adults and even adolescents are prone to be influenced by media trends urging people to undergo cosmetic procedures, so the correlation between age and the interest in plastic surgery is to be further clarified. Moreover, future studies should conduct a survey concerning men's and women's interest in plastic surgery.

Our review has also showed that BMI and general health status are correlated with motivation to seek cosmetic surgery. For example, one could argue that obese men are interested in liposuction or correction of gynaecomastia, but if fit men are interested in such procedures to obtain the perfect appearance, body dysmorphic syndrome could be diagnosed among them. Consequently, these people's body characteristics are a research field, which has to be thoroughly examined. Smoking and alcohol consumption are habits, which are not widely clarified regarding their correlation to the interest in plastic surgery and this is important to be done, given the fact that they may be the direct causes of complications of surgery or anaesthesia.

It is not unusual that people with problematic interpersonal relationships seek a cosmetic procedure, in an attempt to improve their family status or personal relationship, and these patients are seldom satisfied with the surgical results. Our review's results are consistent with this theory, proving that bad relations with parents, single marital status and multiple sexual partners are positive predictors of an interest in plastic surgery.

Our review also highlighted the importance of social norms in relation to cosmetic surgery. In specific, our results show that knowing someone who had a plastic surgery and teasing history are two strong independent variables which predict someone's will to seek a cosmetic procedure. 
It is a common belief that most patients seeking cosmetic surgery aim to improve their psychosocial well-being. Nevertheless, the idea that psychopathology is very common in such patients is outdated. ${ }^{[8,23]}$ Our review showed certain psychopathological symptoms predicting an interest in cosmetic surgery. Even though body dysmorphic disorder patients tend to seek plastic surgery to improve a minor defect, they are seldom satisfied with the result. Hence, they have to be seriously screened during the initial consultation. ${ }^{[24]}$

In terms of personality traits, low self-monitoring, agreeability, openness, conscientiousness and emotional stability were all found to be negative predictors for an interest in plastic surgery. Similarly, high body orientation, indicating people who impose great significance to their image, is a positive predictive factor for someone to seek plastic surgery, while body image evaluation is a negative predictor. Interestingly, low self-esteem has not been found to correlate with this, even though it is strongly correlated with body image evaluation. ${ }^{[16,17]}$

The type of aesthetic operation someone is interested in may be indicative of their psychosocial characteristics. For example, literature indicates an increased risk of suicide among women with breast implants. Indeed, a systematic review of studies addressing cosmetic breast augmentation and suicide concludes a 2-3 times increased risk of suicide for these patients ${ }^{[25}$ and this tendency may be explained through the characteristics of the women who are interested in breast augmentation. ${ }^{[26]}$ Similarly, there is a lot of evidence concerning patients who seek rhinoplasty suggesting that this is a group of patients with a great deal of psychopathology. In addition, a study of facelift patients older than 50 years has found that $90 \%$ had lost an important person in the 5 years before surgery. ${ }^{[27]}$

Our study systematically reviewed the factors that predict someone's interest in cosmetic surgery. This is clinically relevant for plastic surgery surgeons because knowing these psychosocial characteristics can help them understand their patients' motivation.

The next step of this qualitative research field is to examine how psychosocial characteristics, which in fact predict interest in cosmetic surgery, correlate with patients' satisfaction or dissatisfaction with the cosmetic result or the post-operative benefit in their quality of life.
Finally, this systematic review revealed several factors which have never been studied regarding their role in the interest in cosmetic surgery. Future studies should be conducted to examine how status of employment and specific professions, financial status or psychological characteristics relative to work environment, such as burnout syndrome correlate with this interest.

\section{CONCLUSIONS}

This review examined the psychosocial characteristics associated with an interest in cosmetic surgery. As a result, it provides a complete knowledge over this scientific field to plastic surgery surgeons and reveals gaps in literature for future researchers. Understanding cosmetic patients' characteristics, motivation and expectation for surgery is an important aspect of their clinical care to identify those patients more likely to benefit most from the procedure.

\section{Financial support and sponsorship}

Nil.

\section{Conflicts of interest}

There are no conflicts of interest.

\section{REFERENCES}

1. Walker ME, Patel E, Alcon A, Pavre S, Shah A, Ng R, et al. A 12-year analysis of the relationship between market trends and cosmetic case volume. Plast Reconstr Surg 2014;134:43-4.

2. American Society of Plastic Surgeons. Complete Plastic Surgery Statistics Report; 2014. Available from: https:// www. plasticsurgery.org/news/plastic-surgery-statistics?sub= 2014+Plastic+Surgery+Statistics\#section-title.

3. Malick F, Howard J, Koo J. Understanding the psychology of the cosmetic patients. Dermatol Ther 2008;21:47-53.

4. The Cochrane Collaboration. Oxford: The Cochrane Collaboration; Available from: http://www.prisma-statement.org/ PRISMAStatement/PRISMAStatement.aspx.

5. PRISMA Statement; 2014. Available from: http://www. prisma-statement.org/PRISMAStatement/PRISMAStatement. aspx.

6. Oxman AD. Checklists for review articles. BMJ 1994;309:648-51.

7. Littell JH, Corcoran J, Pillai VK. Systematic Reviews and Meta-Analysis. Oxford: Oxford University Press; 2008.

8. Wright MR, Wright WK. A psychological study of patients undergoing cosmetic surgery. Arch Otolaryngol 1975; 101:145-51.

9. Napoleon A. The presentation of personalities in plastic surgery. Ann Plast Surg 1993;31:193-208.

10. Dunofsky M. Psychological characteristics of women who undergo single and multiple cosmetic surgeries. Ann Plast Surg 1997;39:223-8.

11. Cook LS, Daling JR, Voigt LF, deHart MP, Malone KE, Stanford JL, et al. Characteristics of women with and without breast augmentation. JAMA 1997;277:1612-7. 
12. Pertschuk MJ, Sarwer DB, Wadden TA, Whitaker LA. Body image dissatisfaction in male cosmetic surgery patients. Aesthetic Plast Surg 1998;22:20-4.

13. Ishigooka J, Iwao $M$, Suzuki M, Fukuyama $Y$, Murasaki $M$, Miura S. Demographic features of patients seeking cosmetic surgery. Psychiatry Clin Neurosci 1998;52:283-7.

14. Didie ER, Sarwer DB. Factors that influence the decision to undergo cosmetic breast augmentation surgery. J Womens Health (Larchmt) 2003;12:241-53.

15. Sarwer DB, LaRossa D, Bartlett SP, Low DW, Bucky LP, Whitaker LA. Body image concerns of breast augmentation patients. Plast Reconstr Surg 2003;112:83-90.

16. Ferraro GA, Rossano F, D'Andrea F. Self-perception and self-esteem of patients seeking cosmetic surgery. Aesthetic Plast Surg 2005;29:184-9.

17. von Soest T, Kvalem IL, Skolleborg KC, Roald HE. Psychosocial factors predicting the motivation to undergo cosmetic surgery. Plast Reconstr Surg 2006;117:51-62.

18. Frederick DA, Lever J, Peplau LA. Interest in cosmetic surgery and body image: Views of men and women across the lifespan. Plast Reconstr Surg 2007;120:1407-15.

19. Javo IM, Sørlie T. Psychosocial predictors of an interest in cosmetic surgery among young Norwegian women: A population-based study. Plast Reconstr Surg 2009;124:2142-8.
20. Snyder M. Public Appearances, Private Realities: The Psychology of Self-Monitoring. New York: W.H. Freeman; 1986.

21. Gangestad SW, Snyder M. Self-monitoring: Appraisal and reappraisal. Psychol Bull 2000;126:530-55.

22. Chauhan N, Warner JP, Adamson PA. Perceived age change after aesthetic facial surgical procedures quantifying outcomes of aging face surgery. Arch Facial Plast Surg 2012;14:258-62.

23. Baker TJ. Patient selection and psychological evaluation. Clin Plast Surg 1978;5:3-14.

24. Gipson M, Connolly FH. The incidence of schizophrenia and severe psychological disorders in patients 10 years after cosmetic rhinoplasty. Br J Plast Surg 1975;28:155-9.

25. Manoloudakis N, Labiris G, Karakitsou N, Kim JB, Sheena Y, Niakas D. Characteristics of women who have had cosmetic breast implants that could be associated with increased suicide risk: A systematic review, proposing a suicide prevention model. Arch Plast Surg 2015;42:131-42.

26. McLaughlin JK, Wise TN, Lipworth L. Increased risk of suicide among patients with breast implants: Do the epidemiologic data support psychiatric consultation? Psychosomatics 2004;45:277-80.

27. Webb WL Jr., Slaughter R, Meyer E, Edgerton M. Mechanisms of psychosocial adjustment in patients seeking "face-lift" operation. Psychosom Med 1965;27:183-92. 